\title{
The effect of modulating the quantity of enzymes in a model ethanol pathway on metabolic flux in Synechocystis sp. PCC 6803
}

\author{
Paulina Bartasun ${ }^{1}$, Nicole Prandi ${ }^{1}$, Marko Storch ${ }^{1,2}$, Yarin Aknin ${ }^{1,3}$, Mark Bennett ${ }^{1}$, Arianna Palma ${ }^{1}$, Geoff \\ Baldwin $^{1,2}$, Yumiko Sakuragi ${ }^{4}$, Patrik R Jones ${ }^{\text {Corresp., } 1,2}$, John Rowland ${ }^{1}$ \\ ${ }^{1}$ Department of Life Sciences, Imperial College London, London, United Kingdom \\ 2 Imperial College Centre for Synthetic Biology, Imperial College London, London, United Kingdom \\ 3 Institute of Plant Sciences and Genetics in Agriculture, Hebrew University of Jerusalem, Rehovot, Israel \\ 4 Department of Plant and Environmental Sciences, University of Copenhagen, Frederiksberg, Denmark \\ Corresponding Author: Patrik R Jones \\ Email address: p.jones@imperial.ac.uk
}

Synthetic metabolism allows new metabolic capabilities to be introduced into strains for biotechnology applications. Such engineered metabolic pathways are unlikely to function optimally as initially designed and native metabolism may not efficiently support the introduced pathway without further intervention. To develop our understanding of optimal metabolic engineering strategies, a two-enzyme ethanol pathway consisting of pyruvate decarboxylase and acetaldehyde reductase was introduced into Synechocystis sp. PCC 6803. We characteriseda new set of ribosome binding site sequences in Synechocystis sp. PCC 6803 providing a range of translation strengths for different genes under test. The effect of ribosome-bindingsite sequence, operon design and modifications to native metabolism on pathway flux was analysed by HPLC. The accumulation of all introduced proteins was also quantified using selected reaction monitoring mass spectrometry. Pathway productivity was more strongly dependent on the accumulation of pyruvate decarboxylase than acetaldehyde reductase. In fact, abolishment of reductase overexpression resulted in the greatest ethanol productivity, most likely because strains harbouringsingle-gene constructs accumulated more pyruvate decarboxylase than strains carrying any of the multi-gene constructs. Overall, several lessons were learned. Firstly, the expression level of the first gene in anyoperon influenced the expression level of subsequent genes, demonstrating that translational coupling can also occur in cyanobacteria. Longer operons resulted in lower protein abundance for proximallyencoded cistrons. And, implementation of metabolic engineering strategies that have previously been shown to enhance the growth or yield of pyruvate dependent products, through co-expression with pyruvate kinase and/or fructose-1,6- 
greater control over growth and metabolic flux under the tested conditions. 
1 The effect of modulating the quantity of enzymes in a model ethanol pathway on metabolic

2 flux in Synechocystis sp. PCC 6803

3

4 Paulina Bartasun ${ }^{1}$, Nicole Prandi ${ }^{1}$, Marko Storch ${ }^{1}{ }^{4}$, Yarin Aknin ${ }^{2}$, Mark Bennett ${ }^{1}$, Arianna Palma ${ }^{1}$, Geoff S.

5 Baldwin ${ }^{1,4}$, Yumiko Sakuragi ${ }^{3}$, Patrik R. Jones ${ }^{1,4 *}$, John G. Rowland ${ }^{1}$

6

7 1) Department of Life Sciences, Imperial College London, South Kensington Campus, SW7 2AZ London, UK

8 2) Institute of Plant Sciences and Genetics in Agriculture, Hebrew University of Jerusalem, Rehovot, Israel

9 3) Department of Plant and Environmental Sciences, University of Copenhagen, Thorvaldsensvej 40,

10 Frederiksberg DK-1871, Denmark

11 4) Imperial College Centre for Synthetic Biology, Imperial College London, South Kensington Campus, SW7

12 2AZ London, UK

13

14

15

*corresponding author: p.jones@imperial.ac.uk

16

17 Keywords: cyanobacteria, ethanol, optimization, metabolic engineering 


\section{Abstract}

19 Synthetic metabolism allows new metabolic capabilities to be introduced into strains for biotechnology applications. Such engineered metabolic pathways are unlikely to function optimally as initially designed and native metabolism may not efficiently support the introduced pathway without further intervention. To develop our understanding of optimal metabolic engineering strategies, a two-enzyme ethanol pathway consisting of pyruvate decarboxylase and acetaldehyde reductase was introduced into Synechocystis sp. PCC 6803. We characterised a new set of ribosome binding site sequences in Synechocystis sp. PCC 6803 providing a range of translation strengths for different genes under test. The effect of ribosome-binding site sequence, operon design and modifications to native metabolism on pathway flux was analysed by HPLC. The accumulation of all introduced proteins was also quantified using selected reaction monitoring mass spectrometry. Pathway productivity was more strongly dependent on the accumulation of pyruvate decarboxylase than acetaldehyde reductase. In fact, abolishment of reductase over-expression resulted in the greatest ethanol productivity, most likely because strains harbouring single-gene constructs accumulated more pyruvate decarboxylase than strains carrying any of the multi-gene constructs. Overall, several lessons were learned. Firstly, the expression level of the first gene in any operon influenced the expression level of subsequent genes, demonstrating that translational coupling can also occur in cyanobacteria. Longer operons resulted in lower protein abundance for proximally-encoded cistrons. And, implementation of metabolic engineering strategies that have previously been shown to enhance the growth or yield of pyruvate dependent products, through coexpression with pyruvate kinase and/or fructose-1,6-bisphosphatase/sedoheptulose-1,7-bisphosphatase, indicated that other factors had greater control over growth and metabolic flux under the tested conditions. 


\section{Introduction}

Synthetic metabolism is the creation of novel metabolism achieved by the combination of enzymes and regulatory elements that in most cases have not co-evolved. Although this opens up new opportunities for biotechnological solutions (Erb et al. 2017) such synthetic pathways are unlikely to function optimally due to sub-optimal balance of enzyme abundance and/or regulation of expression. For example, Zelcbuch et al. (2013) clearly showed the impact of variation in protein expression on flux through a complex synthetic pathway. Several factors influence the accumulation of proteins, including choice of promoter, ribosome binding site (RBS), gene copy number and operon structure. Furthermore, the stability of transcripts and the rate of protein degradation will naturally vary and can also be engineered (Zelcbuch et al. 2013).

The field of cyanobacteria-based synthetic biology has recently been attracting significant attention. However, compared to E. coli, studies on synthetic pathway optimization are scarce in cyanobacteria. Several review articles have presented recent advancements and discussed obstacles in the development of tools for better control of protein expression (Angermayr et al. 2015; Carroll et al. 2018; Gao et al. 2016; Lai \& Lan 2015; Ramey et al. 2015; Wang et al. 2012; Yu et al. 2013). Most attention on the genetic parts used to engineer cyanobacteria has so far been directed toward the optimal choice of promoter (Guerrero et al. 2012; Huang et al. 2010; Markley et al. 2015; Qi et al. 2013; Ruffing et al. 2016) or RBS sequence (Englund et al. 2016; Heidorn et al. 2011; Oliver et al. 2014; Veetil et al. 2017; Wang et al. 2018; Yunus \& Jones 2018). The impact of gene order in a cyanobacterial synthetic operon has also been investigated (Nozzi \& Atsumi 2015). Other approaches have included the development of broad-host range expression systems for cyanobacteria (Taton et al. 2014) and evaluation of integration at different genomic loci vs. episomal expression (Angermayr et al. 2014; Guerrero et al. 2012). Despite these efforts, we are not aware of any prior studies that have directly quantified heterologously expressed proteins in cyanobacterial hosts. Hence, there is still limited data to determine cause-and-effect relationships in previous optimization studies.

In the present work we have focused on optimizing an engineered ethanol pathway introduced into Synechocystis sp. PCC 6803 via a chromosomally integrated bicistronic artificial operon. We evaluated the impact of variant RBS strengths within the operon, the choice of operon design, and previously reported modifications to native metabolism on product yield. The strains were characterized by direct measurements of both product and enzyme concentrations. Although no major advancements in product yield were observed, the study contributes to our understanding of factors that influence the outcome of metabolic engineering designs in cyanobacteria. 
METHODS

\section{Culture conditions}

The glucose-tolerant wild-type strain of Synechocystis sp. PCC 6803 (provided by Dr. Patricia Armshaw, UL, Limerick, Ireland) and transformed strains thereof were were cultured in BG-11 medium (Stanier et al. 1971). Cultures were grown in $100 \mathrm{~mL}$ Erlenmeyer flasks containing $20 \mathrm{~mL}$ of BG-11 at $30^{\circ} \mathrm{C}$ in a Photon Systems Instruments AlgaeTron230 growth chamber having $1 \%(\mathrm{v} / \mathrm{v}) \mathrm{CO}_{2}$ (using Ecotechnics (UK) $\mathrm{CO}_{2}$ sensor and controller) and a light intensity setting of $60 \mu \mathrm{mol}$ photons $/ \mathrm{m}^{2} / \mathrm{s}$ (cool-white LED with added far-red LEDs 735 nM). The orbital Unimax 1010 (Heidolph Instruments) shaker was set to shake at a speed of $210 \mathrm{rpm}$. Cultures were supplemented with spectinomycin (50 $\mathrm{gg} / \mathrm{mL}$ final concentration) and IPTG (1mM final concentration) when appropriate. Growth was monitored by following optical density (OD) via absorbance at $730 \mathrm{~nm}$ (Infinite 200 Pro spectrophotometer, Tecan Group Ltd. Switzerland). E.coli strains were grown at $37^{\circ} \mathrm{C}$ in Luria-Broth medium supplemented with antibiotic when necessary at the following final concentrations: spectinomycin $50 \mu \mathrm{g} / \mathrm{mL}$, kanamycin $50 \mu \mathrm{g} / \mathrm{mL}$ or ampicillin $100 \mu \mathrm{g} / \mathrm{mL}$.

\section{DNA constructs}

All DNA manipulations were performed using BASIC DNA assembly (Storch et al. 2015) and plasmids transformed and propagated in E. coli DH5 alpha. Briefly, DNA parts used for the assembly were amplified using primers containing BASIC Prefix and Suffix and cloned into PJET1.2 vector. The primers used are listed in Supplementary Table 1. The DNA constructs were assembled using Bsal-digested pJET-storage vectors and appropriate linkers as described in (Storch et al. 2015). BASIC linkers that encoded five different RBS sequences (Supplementary Table 2) were used to assemble both single gene and operon constructs of varying translational strength. Supplementary Table 3 contains a list of plasmids constructed in this study.

\section{Synechocystis sp. PCC 6803 transformation}

Transformation of Synechocystis sp. PCC 6803 with plasmid DNA constructs was performed as described in (Armshaw et al. 2015) except for the use of glucose. Spectinomycin at a final concentration of $50 \mu \mathrm{g} / \mathrm{mL}$ was used as a selection marker. Chromosomal integration and full segregation were verified by PCR and Sanger sequencing of the obtained amplification products (Source BioScience, UK).

\section{Fluorescence measurements}


103 For eYFP measurements cells were grown up as described previously to OD $_{730 \mathrm{~nm}}$ of 0.5 and IPTG was added

104 to a final concentration of $1 \mathrm{mM}$. After 7 days, the cultures were diluted 10 times and absorbance and 105 fluorescence readings were taken using the Infinite 200 Pro spectrophotometer (absorption: 730 nm; 106 excitation $503 \mathrm{~nm}$, emission $540 \mathrm{~nm}$ ). The fluorescence was normalized against wild-type strain control 107 and cell density.

108 Ethanol measurements

109 Cells were grown up to OD $730 \mathrm{~nm}$ of 0.5 and IPTG was added to final concentration of $1 \mathrm{mM}$. After 7 days, 1 $110 \mathrm{~mL}$ of every culture was taken, the cells were spun down $(4000 \mathrm{~g}, 10 \mathrm{~min})$ and the ethanol content of the 111 supernatant quantified by HPLC (1200 series, Agilent Technologies, Böblingen, Germany). A prepacked 112 HPLC carbohydrate analysis AMINEX HPX-87H column (300 x 7.8 mm, hydrogen form, $9 \mu \mathrm{m}$ particle size, $1138 \%$ cross-linkage) with industrial grade guard cartridge (30 x $4.6 \mathrm{~mm}$, hydrogen form) (Biorad, USA) was 114 heated up to $60^{\circ} \mathrm{C}$. The mobile phase was $5 \mathrm{mM}$ sulphuric acid in water (HPLC grade) with a flow rate of $1150.6 \mathrm{~mL} / \mathrm{min}$. The signals were acquired with a refractive index detector. A standard curve made up of serial 116 dilutions of absolute ethanol (VWR) was used to determine the quantity of ethanol in each sample.

\section{Sample preparation for SRM Mass spectrometry analysis}

118 Cells were grown up to $\mathrm{OD}_{730 \mathrm{~nm}}$ of 0.5 and IPTG was added to final concentration of 1 mM. After 7 days, $11915 \mathrm{~mL}$ of cultures were harvested by centrifugation ( $4000 \mathrm{~g}, 30 \mathrm{~min}$ ). The pellets were re-suspended in 1 $120 \mathrm{~mL}$ of extraction buffer (20 mM Tris- $\mathrm{HCl}$ pH 8.0, 1 mM EDTA, 2 mM DTT). Five hundred microliters of cell suspension were mixed with $500 \mu \mathrm{L}$ of extraction buffer and washed glass beads (Sigma) in a $2 \mathrm{~mL}$ lysis tube. The cells were disrupted for 10 minutes at $30 \mathrm{~Hz}$ using the TissueLyser II (Qiagen, USA). To remove unbroken cells from the lysate the samples were spun down at $12000 \mathrm{~g}$ for $10 \mathrm{~min}$. Protein concentration in the supernatant was estimated using the DC Protein Assay (Biorad, USA). Next, $100 \mu \mathrm{g}$ of protein was reduced with $10 \mathrm{mM}$ DTT in $50 \mathrm{mM}$ ammonium bicarbonate (final concentrations) for $1 \mathrm{hr}$ at $56^{\circ} \mathrm{C}$, shaking followed by alkylation with $50 \mathrm{mM}$ iodoacetamide (final concentration) for 30 mins at $37^{\circ} \mathrm{C}$ in the dark, 500 rpm (Eppendorf thermomixer). Proteomics-grade trypsin (Promega, USA) was added to the protein sample in a 1:50 mass ratio and the samples were incubated for about $4 \mathrm{~h}$ at $37^{\circ} \mathrm{C}(700 \mathrm{rpm}$, Eppendorf thermomixer). Subsequently, trypsin was added to the samples again in the same ratio and the samples were incubated at $37^{\circ} \mathrm{C}$ (700 rpm, Eppendorf thermomixer, Germany) for a further 16 hrs to ensure full digestion of the proteins. The digestion was then stopped by lowering the pH to less than 2 by adding

132 formic acid and incubating for $30 \mathrm{~min}$ at $37^{\circ} \mathrm{C}, 500 \mathrm{rpm}$. Sample clarification and removal of water- 
133 immiscible products were achieved by centrifugation for $10 \mathrm{~min}$ at $12000 \mathrm{~g}$. Supernatants were stored at $134-80^{\circ} \mathrm{C}$ until further SRM analysis.

\section{Analysis of protein by LC-MS/MS}

136 The LC-MS/MS comprised of an Agilent 1100 LC system and an ABSciex 6500 Qtrap MS. Chromatography 137 was performed on a Phenomenex Luna C18(2) column (100mm $\times 2 \mathrm{~mm} \times 3 \mu \mathrm{m})$ at a temperature of $50^{\circ} \mathrm{C}$ 138 utilising a gradient solvent system of $\mathrm{A}\left(94.9 \% \mathrm{H}_{2} \mathrm{O}, 5 \% \mathrm{CH}_{3} \mathrm{CN}, 0.1 \%\right.$ formic acid,) and $\mathrm{B}\left(94.9 \% \mathrm{CH}_{3} \mathrm{CN}, 5 \%\right.$ $139 \mathrm{H}_{2} \mathrm{O}, 0.1 \%$ formic acid). A gradient from $0 \% \mathrm{~B}$ to $35 \% \mathrm{~B}$ over 30 minutes at a flow rate of $250 \mathrm{~mL} / \mathrm{min}$ was 140 used. The column was then washed with 100\% B for 3 min and then re-equilibrated with 100\% A for 6 141 minutes. Typically $40 \mu \mathrm{L}$ injections were used for the analysis.

142 The MS was configured with an Ion Drive Turbo V source, Gas 1 and 2 were set to 40 and 60 respectively, 143 the source temperature to $500^{\circ} \mathrm{C}$ and the ion spray voltage to $5500 \mathrm{~V}$.

144 The MS, configured with high mass enabled, was used in "Trap" mode to acquire Enhanced Product lon 145 (EPI) scans for peptide sequencing and "Triple Quadruple" mode for Multiple Reaction Monitoring. Data 146 were acquired and analysed using SCIEX software Analyst 1.6.1 and MultiQuant 3.0.

147 Proteotypic peptides. Signature peptides for pyruvate decarboxylase (Pdc), acetaldehyde reductase (Adh, 148 also sometimes referred to as 'alcohol dehydrogenase'), pyruvate kinase (Pyk), bifunctional fructose-1,6149 bisphosphatase/sedoheptulose-1,7-bisphosphatase (Bibp) and AtpB protein (SIr1329) were determined 150 from trial MRM-MS runs. AtpB was used as an internal standard for protein normalisation as it exhibited

151 minimal fluctuation across treatments in a previous study (Vuorijoki et al. 2016). The typical work flow to 152 select the best signature peptides consisted of samples analysis using transitions generated by an in silico 153 analysis using Skyline (MacLean et al. 2010). The identity of candidate peptides was then confirmed by 154 Enhanced Product Ion scans. Synechocystis sp. PCC 6803 background proteome 155 (http://genome.kazusa.or.jp/cyanobase) was used to confirm the uniqueness.

156 Typically 3-5 transitions per peptide were used. In the final method 2-4 peptides per proteins were used 157 for its identification and quantification. Signature peptides for each protein are listed in Supplementary 158 Table 4. Protein quantification was performed based on relative peak intensities of the analysed protein 159 and normalized to the relative peak intensities of the AtpB native standard.

161 Statistical Analysis

162 For all statistical analysis with samples taken from microbial cultures, a normal distribution was 163 assumed, as discussed in Fay and Gerow (2013). Three biological replicates were employed for every 
164 treatment and/or condition. One-way ANOVA was carried out using the one-way ANOVA with post-hoc

165 Tukey HSD (Honestly Significant Difference) test calculator prepared by Navendu Vasavada

166 (http://astatsa.com/OneWay_Anova_with_TukeyHSD/). For the analysis where two factors (RBS in front

167 of PDC, RBS in front of ADH) were evaluated in a combinatorial design we employed the anova2 and

168 multcompare functions of Matlab R2017b. An Excel template provided by Weissgerberger et al.

169 (Weissgerber et al. 2015) was modified and used for all scatterplots. 


\section{RESULTS AND DISCUSSION}

171 Operon architectures, ubiquitous features of bacterial genomes, are an effective and highly efficient 172 means for driving coordinated production of proteins involved in metabolic pathways. Operons are 173 therefore also highly attractive for the synthetic implementation of entire heterologous metabolic 174 pathways to enable bioproduction of target compounds (Akhtar \& Jones 2008). We were interested in 175 understanding further what the best design for such operons may be when used in cyanobacteria.

176 For this purpose we used a well-studied and simple system, pyruvate-derived ethanol biosynthesis (Deng 177 \& Coleman 1999), as a model two-protein metabolic production system. Principles derived from this 178 simple model may be applicable to other, more complex bioproduction systems. Many other factors than 179 pathway composition will also influence the metabolic outcome under laboratory conditions, including 180 light and gas exchange, and when the conditions are sub-optimal this may limit the impact of 181 improvement in any pathway. For the sake of simplicity, the focus in the present study was placed on a 182 relative comparison employing only modulation in pathway proteins as experimental treatments.

\section{Selected RBS variants are functional in vivo and display a wide range of protein expression strength}

A total of 5 RBS variants (Supplementary Table 2) were selected for characterisation via assays of fluorescence emitted by RBS-eyfp constructs. Four RBS sequences have been characterised previously in E. coli, while the fifth is specific to the anti-Shine-Dalgarno sequence of the Synechocystis sp. PCC 6803 ribosome (Heidorn et al. 2011). These sequence variants were contained within linker sequences ("RBS linkers") used in the BASIC DNA construct assembly process (Storch et al. 2015).

Five constructs were generated for PA1lacO1 promoter-driven expression of eYFP containing one of five 5'-proximal RBS variants (Supplementary Table 3). The PA1lacO1 promoter was previously shown to be inducible with IPTG in Synechocystis sp. PCC 6803 (Guerrero et al. 2012). All ethanol pathway encoding constructs were designed for chromosomal integration at the well-studied s/r0168 neutral site (Angermayr et al. 2012).

All five constructs were introduced into Synechocystis sp. PCC 6803 and fully segregated mutants were isolated. The fluorescence of the cultures was measured following a 7-day induction period in the presence of IPTG (Figure 1). RBS A, which has been reported as weak in E. coli (Salis et al. 2009), turned out to also show minimal expression in the cyanobacterial host strain. The commonly used RBS elements in iGEM projects, RBS B (BBa_B0064) and RBS C (BBa_B0034), displayed intermediate strength. RBS E, which has been chosen as a cyanobacteria-specific RBS, enabled the highest expression of all tested 
202 relative strength order in cyanobacteria: RBSA $<$ RBS B $<$ RBS D $<$ RBS $C<R B S E$. This set of RBS sequences

203 satisfied the main objectives with this initial screening, to be capable of modulating protein abundance 204 across a broad range in cyanobacteria.

205

206 Creation of a combinatorial library of ethanologenic strains.

207 We wanted to evaluate how the expression and organisation of the $p d c$ and $a d h$ genes in an operon would 208 impact both flux and product formation through the synthetic ethanol pathway. Further, we wanted to 209 correlate the output to protein levels and understand the impact on growth. To achieve this we designed 210 an operon matrix with varying levels of expression of the two genetic components governed by the 211 characterised RBS linker sequences used in the construction. In total, 25 constructs were created with all 212 combinations of RBS sequences upstream of the $p d c$ and adh genes. Promoter, terminator and genomic 213 integration locus were the same as for the eYFP constructs described above. Twenty-four out of the 25 214 constructs were successfully used to transform Synechocystis followed by segregation through serial 215 passage and confirmation by PCR. These mutants were then used to test ethanol production efficiency 216 and quantify the abundance of the pathway components for each combination of RBS variants. The mutant containing RBS D upstream of $p d c$ and RBS A upstream of adh could not be segregated despite repeated attempts.

\section{RBS variation results in substantial differences in ethanol production.}

The accumulation of ethanol after 7 days of cultivation was assessed by HPLC. As expected, variation in ethanol production was observed among the tested strains (Figure 2). The strains exhibited minor differences in growth curves and final optical density values with the exception of strains harboring RBS B for PDC and RBS C for ADH (Supplementary Data 2). Ethanol production was strongly dependent on the RBS sequences for the $p d c$ gene as significant variation in ethanol production was observed between strains harboring different RBS upstream of $p d c$ while having the same RBS upstream of adh. No such trend was observed for variation of the RBS upstream of adh, when the pdc RBS was held constant. Interestingly, RBS A placed upstream of $p d c$ led to higher ethanol production than RBS B upstream of $p d c$, even though RBS A was the weakest RBS in the context of eyfp. RBS D, which was considered weaker than RBS C when tested with the eyfp gene, provided a higher level of ethanol when placed upstream of $p d c$ than RBS C. However, such differences in RBS activity are not surprising as it is well known that the 
233

234

235

236

237

238

239

240

241

242

243

244

245

246

247

248

249

250

251

252

253

254

255

256

257

258

259

260

261

262

263

sequence context (Salis et al. 2009). Therefore, we cannot assume that changes in RBS will lead to the same variation in protein abundance when coupled with $p d c$ or adh as compared to eyfp.

\section{RBS variants modulate protein abundance.}

To understand the cause-and-effect relationships better, we analysed the abundance of Pdc and Adh in our ethanologenic strains via selected reaction monitoring (SRM) on an LC-MS/MS (Figure 3), assuming that each internal enzyme activity at least to some degree correlated with the measured quantity of each protein. Protein abundances were normalised to the abundance of the ATP synthase beta subunit (AtpB, s/r1329). From these data, it is clear that RBS strengths can be ranked in the following order: RBS A $\leq$ RBS $B<$ RBS C $<$ RBS D $<$ RBS E. This relationship holds for both Pdc (Figure 3A) and Adh (Figure 3B). When a weak RBS (RBS A or RBS B) was placed upstream of $p d c$, regardless of the RBS present upstream of $a d h$, both the ethanol yield and relative abundance of Pdc were low. With increasing $p d c$ RBS strength the production of ethanol and the accumulation of both Pdc and Adh all increased. The strength of the RBS upstream of $a d h$, while capable of modulating Adh accumulation, had negligible influence on the abundance of Pdc.

Next, we calculated the correlation between enzyme abundance and ethanol yield and also compared how the abundance of one enzyme correlated with the other. The correlation between protein abundance and ethanol yield was strongest for Pdc $\left(R^{2}=0.89\right.$; Figure $\left.4 A\right)$, while for Adh this correlation was much weaker $\left(R^{2}=0.44\right.$; Figure $4 B$ ). Comparing protein levels, Adh abundance was, although weak, slightly more strongly correlated with Pdc abundance $\left(R^{2}=0.51\right)($ Supplementary Data 2, Fig. 4 sheet). Together, these observations indicate the operation of "translational coupling" (Levin-Karp et al. 2013) between high-level Pdc expression and Adh.

\section{Enhanced Pdc expression is sufficient for yield-improvement.}

The weak correlation between Adh and ethanol titre suggested that Adh over-expression was not necessary. In order to test this hypothesis, five new strains were constructed that were similar to the previous 24 strains except that they lacked the adh gene. Once again, five different RBS sequences were placed upstream of $p d c$. The constructs were transformed into Synechocystis 6803 and fully segregated mutants were obtained for all combinations. Ethanol production and protein abundance were tested, with five of the previously constructed ethanol-producing strains used as controls ( $A A, B B, C C, D D, E E)$. Ethanol yields were, greater for the monocistronic (Pdc-only) strains compared with the equivalent RBS bicistronic 
264 (Pdc-Adh) strains, when comparing C, D and E with CC, DD and EE, respectively (Figure 5A). As expected,

265 the Pdc protein abundance was also greater in three out of five mono- vs. double-cistronic comparisons

266 (i.e. A, D, E all had increased Pdc content compared to AA, DD and EE, respectively; Figure 5B). These

267 observations are consistent with either expression from a shorter transcript being more efficient and/or

268 that there is a reduced metabolic burden placed on the cell as fewer resources are required to express

269 the single protein. Alternatively, the new transcripts may have differed in their secondary structure. Lim

270 et al. (Lim et al. 2011) have reported that, in E. coli, proteins encoded on long operons are more highly

271 expressed. This is in contrast to what we observed in cyanobacteria with proteins encoded on longer

272 operons being poorly expressed. Pdc abundance was once again linearly correlated with ethanol yield

273 (Figure 5C) and can be improved further as there is no evidence of a plateau in ethanol productivity at the

274 protein abundances achieved in these strains.

275

276 Further modulation of host metabolism does not improve product yield.

277 Sustainable commercial production of ethanol using cyanobacteria will require increased productivities

278 and product titres. The strong relationship between ethanol productivity and quantity of pyruvate

279 decarboxylase suggested this was the key limiting factor. However, previous studies have found at least

280 some impact from optimization of native metabolism even when the activity of a single pyruvate-

281 dependent enzyme (lactate dehydrogenase) was clearly rate-limiting (Angermayr et al. 2014). We

282 therefore evaluated the effect of overexpressing pyruvate kinase (Pyk) from E. coli, which should improve

283 the supply of the precursor pyruvate as was previously reported (Angermayr et al. 2014). Another 284 possibility is to over-express BiBP, a bifunctional fructose-1,6-bisphosphatase/sedoheptulose-1,7-

285 bisphosphatase from Synechocystis sp. PCC 6803 (Jiang et al. 2012), which has been reported to improve

286 growth and photosynthetic rate in the cyanobacterium Synechococcus sp. PCC 7002 and Synechocystis sp.

287 PCC 6803 presumably by enhancing flux through central carbon metabolism (De Porcellinis et al. 2018;

288 Liang et al. 2018).

289 To strains already harbouring the pdc-adh cassette with RBS E in front of both genes, we 290 introduced Pyk and BiBP as a second operon at a different genomic locus (s/r1395). The new cassette

291 harbouring Pyk and BiBP was combined with four different RBS elements (A, B, D, E) located upstream of 292 each coding sequence and placed under the control of the constitutive $P_{\text {trc }}$ promoter. Fully segregated 293 mutants were obtained for eight out of sixteen designs. However, analysis of these strains revealed no 294 improvement in ethanol production (Figure 6A) compared to the original strain, despite detectable 295 increases in both Pyk and BiBP abundance (Figure 6B). In an earlier study with Synechococcus sp. PCC 
296

297

298

299

300

301

302

303

304

305

306

307

308

309

310

311

312

313

314

315

316

317

318

319

320

321

322

323

324

325

326

7002, over-expresison of BiBP resulted in a growth benefit dependent on both high light and high carbon availability (De Porcellinis et al. 2018). In the present study, the over-expression of BiBP had no or a negative impact on growth (Figure $6 \mathrm{C}$ ). However, the environmental conditions, the engineering designs and the host strain were different in the two studies, thereby ruling out any possibility to compare between them. Nevertheless, the increase in Pyk and BiBP abundance correlated well with the strength of the RBS used. Again we observed that the increased expression level of the protein encoded first in the operon (pyk) influenced the expression of the protein encoded last (Bibp). For example, this can be seen if we compare the expression of Pyk and BiBP in strains EE_AD \& EE_DD.

\section{New insights were uncovered and several challenges remain to be addressed}

All DNA constructs used in this study were generated via BASIC methodology (Storch et al. 2015). This method enabled quick and reliable generation of a series of DNA constructs. The main advantage of BASIC is its modularity, where a DNA part once cloned into a storage vector can be used in various assemblies without any further need for amplification. It proved extremely useful in our work involving the preparation of a large number of molecular constructs with different genetic parts, including small RBS elements. In overlap assembly techniques like Gibson (Gibson et al. 2009) such work would involve amplification of each gene with different primers for each construct in order to introduce the RBS sequence and compatible overhangs. Whereas when using BASIC the order of assembly is directed by the employed linkers which may also contain the RBS sequence, for example. Furthermore, in contrast to Golden Gate based approaches, the single-tier BASIC formatted parts can be assembled in any order and in varying numbers without reformatting. The approach we took significantly reduced the workload and enabled us to generate a large number of the required molecular constructs in a very short time. The bottleneck in strain construction was instead shifted to cyanobacteria transformation and segregation. For example, we were unable to isolate a strain containing RBS D upstream of $p d c$ and RBS A upstream of adh. Frequently mooted hypotheses for such observations include metabolic burden of protein overproduction and product or intermediate substrate toxicity. We infer from our data that this strain would not have produced higher levels of protein, ethanol or the intermediate acetaldehyde than other strains in the set. It would appear therefore, that some other underlying phenomenon may be at play.

We could also not isolate any strains for the four-cistron operon designs and several attempts were required to generate strains containing tri-cistronic operons. This suggests that there exists a tradeoff between integration/segregation efficiency and insert length. To the best of our knowledge there have 
327 been no detailed studies performed on the impact of insert length on transformation efficiency in cyanobacteria. Three tri-cistronic operon strains remained only partially segregated, which cannot be confidently attributed to genomic integration locus, burden of protein over-production or substrate / product toxicity. Operon lengths in these cases were identical. The presence of specific sequence features within these constructs and their impact on genome stability cannot be discounted (Jones 2014).

In terms of lessons learned, one of the more striking observations in our data is the evidence for translational coupling. Translational coupling in general is not well characterised, particularly in cyanobacteria. The extent of translational coupling is related to inter-cistronic distance and has been at least partially characterised in other species (Levin-Karp et al. 2013). Despite this being highly relevant for strain design, the distances required to either maximise or abolish translational coupling in cyanobacterial species remains entirely undescribed. This also highlights that, while assembly methods such as BASIC are extremely useful tools for rapid prototyping, other bottlenecks may limit throughput and delivery of fully optimized bioproduction strains.

It has been reported by others that longer operons result in higher protein abundance for proximally-encoded cistrons (Lim et al. 2011). Their results, demonstrated using $E$. coli, are not consistent with the data we present here on Pdc abundance: longer operons result in lower protein abundance for proximally-encoded cistrons in Synechocystis sp. PCC 6803. Whether our observations are specific for the ethanol-cassette design or reflect more general differences between the cyanobacteria and other model bacterial species remains to be investigated.

The work also illustrates the importance of limiting factors for metabolic engineering, as also highlighted in the work of Angermayr and colleagues with lactate (Angermayr \& Hellingwerf 2013). At the enzyme level, the first enzyme of the introduced pathway (Pdc) was clearly limiting flux through the pathway and changes to the second enzyme (Adh) did not have much of an impact. At the whole-cell metabolism level, despite successful over-expression of two enzymes previously demonstrated to stimulate either growth or pyruvate-dependent product yield, no or minimal impact on the ethanol pathway was observed. If possible, identification of the key limiting factor(s) that control most of the metabolic flux through an introduced pathway, should first be pursued. With respect to the ethanol pathway, Pdc was that factor. Future studies should investigate how this activity could be enhanced.

\section{Concluding Remarks.}


357 Using ethanol production as a model system we have learned more about some of the considerations 358 required for implementing optimal synthetic metabolic strain designs in Synechocystis sp. PCC 6803. (1) 359 RBS variation in operon constructs can result in at least a 3.5-fold variation in pathway activity. (2) 360 Translational coupling can have a major influence on the expression of multiple proteins encoded by an 361 operon, e.g. influencing choice of gene order. (3) The presence or absence of downstream genes in an 362 operon (i.e. operon length and/or composition) can also influence the expression of upstream genes. And 363 (4), BASIC is a useful methodology for rapid assembly of construct libraries with diversified composition. 364 
365

366

367

368

369

370

371

372

373

374

375

376

377

378

379

380

381

382

383

384

385

386

387

388

389

390

391

392

393

394

395

\section{References}

Akhtar MK, and Jones PR. 2008. Engineering of a synthetic hydF-hydE-hydG-hydA operon for biohydrogen production. Analytical Biochemistry 373:170-172. 10.1016/j.ab.2007.10.018

Angermayr SA, and Hellingwerf KJ. 2013. On the Use of Metabolic Control Analysis in the Optimization of Cyanobacterial Biosolar Cell Factories. J Phys Chem B. 10.1021/jp4013152

Angermayr SA, Paszota M, and Hellingwerf KJ. 2012. Engineering a Cyanobacterial Cell Factory for Production of Lactic Acid. Applied and Environmental Microbiology 78:7098-7106. 10.1128/Aem.01587-12

Angermayr SA, Rovira AG, and Hellingwerf KJ. 2015. Metabolic engineering of cyanobacteria for the synthesis of commodity products. Trends in Biotechnology 33:352-361. 10.1016/j.tibtech.2015.03.009

Angermayr SA, van der Woude AD, Correddu D, Vreugdenhil A, Verrone V, and Hellingwerf KJ. 2014. Exploring metabolic engineering design principles for the photosynthetic production of lactic acid by Synechocystis sp PCC6803. Biotechnology for Biofuels 7. Artn 99

10.1186/1754-6834-7-99

Armshaw P, Carey D, Sheahan C, and Pembroke JT. 2015. Utilising the native plasmid, pCA2.4, from the cyanobacterium Synechocystis sp strain PCC6803 as a cloning site for enhanced product production. Biotechnology for Biofuels 8. ARTN 201

$10.1186 / \mathrm{s} 13068-015-0385-x$

Carroll AL, Case AE, Zhang A, and Atsumi S. 2018. Metabolic engineering tools in model cyanobacteria. Metabolic Engineering. https://doi.org/10.1016/i.ymben.2018.03.014

De Porcellinis AJ, Norgaard H, Brey LMF, Erstad SM, Jones PR, Heazlewood JL, and Sakuragi Y. 2018. Overexpression of bifunctional fructose-1,6-bisphosphatase/sedoheptulose-1,7-bisphosphatase leads to enhanced photosynthesis and global reprogramming of carbon metabolism in Synechococcus sp PCC 7002. Metabolic Engineering 47:170-183. 10.1016/j.ymben.2018.03.001

Deng M, and Coleman J. 1999. Ethanol synthesis by genetic engineering in cyanobacteria. Appl Environ Microbiol 65:523-528.

Englund E, Liang FY, and Lindberg P. 2016. Evaluation of promoters and ribosome binding sites for biotechnological applications in the unicellular cyanobacterium Synechocystis sp PCC 6803. Scientific Reports 6. ARTN 36640

10.1038/srep36640 
396

Erb TJ, Jones PR, and Bar-Even A. 2017. Synthetic metabolism: metabolic engineering meets enzyme design. Current Opinion in Chemical Biology 37:56-62. 10.1016/j.cbpa.2016.12.023

Gao XY, Sun T, Pei GS, Chen L, and Zhang WW. 2016. Cyanobacterial chassis engineering for enhancing production of biofuels and chemicals. Applied Microbiology and Biotechnology 100:3401-3413. 10.1007/s00253-016-7374-2

Gibson DG, Young L, Chuang RY, Venter JC, Hutchison CA, and Smith HO. 2009. Enzymatic assembly of DNA molecules up to several hundred kilobases. Nature Methods 6:343-U341. 10.1038/Nmeth.1318

Guerrero F, Carbonell V, Cossu M, Correddu D, and Jones PR. 2012. Ethylene Synthesis and Regulated Expression of Recombinant Protein in Synechocystis sp PCC 6803. Plos One 7. ARTN e50470

10.1371/journal.pone.0050470

Heidorn T, Camsund D, Huang HH, Lindberg P, Oliveira P, Stensjo K, and Lindblad P. 2011. Synthetic Biology in Cyanobacteria: Engineering and Analyzing Novel Functions. Methods in Enzymology, Vol 497: Synthetic Biology, Methods for Part/Device Characterization and Chassis Engineering, Pt A 497:539-579. 10.1016/B978-0-12-385075-1.00024-X

Huang HH, Camsund D, Lindblad P, and Heidorn T. 2010. Design and characterization of molecular tools for a Synthetic Biology approach towards developing cyanobacterial biotechnology. Nucleic Acids Research 38:2577-2593. 10.1093/nar/gkq164

Jiang YH, Wang DY, and Wen JF. 2012. The independent prokaryotic origins of eukaryotic fructose-1, 6bisphosphatase and sedoheptulose-1, 7-bisphosphatase and the implications of their origins for the evolution of eukaryotic Calvin cycle. Bmc Evolutionary Biology 12. Artn 208

$10.1186 / 1471-2148-12-208$

Jones PR. 2014. Genetic Instability in Cyanobacteria - An Elephant in the Room? Frontiers in Bioengineering and Biotechnology 2. 10.3389/fbioe.2014.00012

Lai MC, and Lan El. 2015. Advances in Metabolic Engineering of Cyanobacteria for Photosynthetic Biochemical Production. Metabolites 5:636-658. 10.3390/metabo5040636

Levin-Karp A, Barenholz U, Bareia T, Dayagi M, Zelcbuch L, Antonovsky N, Noor E, and Milo R. 2013. Quantifying Translational Coupling in E. coli Synthetic Operons Using RBS Modulation and Fluorescent Reporters. Acs Synthetic Biology 2:327-336. 10.1021/sb400002n

Liang FY, Englund E, Lindberg P, and Lindblad P. 2018. Engineered cyanobacteria with enhanced growth show increased ethanol production and higher biofuel to biomass ratio. Metabolic Engineering 46:51-59. 10.1016/j.ymben.2018.02.006 
428

429

430

431

432

433

Lim HN, Lee Y, and Hussein R. 2011. Fundamental relationship between operon organization and gene expression. Proceedings of the National Academy of Sciences of the United States of America 108:10626-10631. 10.1073/pnas.1105692108

MacLean B, Tomazela DM, Shulman N, Chambers M, Finney GL, Frewen B, Kern R, Tabb DL, Liebler DC, and MacCoss MJ. 2010. Skyline: an open source document editor for creating and analyzing targeted proteomics experiments. Bioinformatics 26:966-968. 10.1093/bioinformatics/btq054

Markley AL, Begemann MB, Clarke RE, Gordon GC, and Pfleger BF. 2015. Synthetic Biology Toolbox for Controlling Gene Expression in the Cyanobacterium Synechococcus sp strain PCC 7002. Acs Synthetic Biology 4:595-603. 10.1021/sb500260k

Nozzi NE, and Atsumi S. 2015. Genome Engineering of the 2,3-Butanediol Biosynthetic Pathway for Tight Regulation in Cyanobacteria. Acs Synthetic Biology 4:1197-1204. 10.1021/acssynbio.5b00057

Oliver JWK, Machado IMP, Yoneda H, and Atsumi S. 2014. Combinatorial optimization of cyanobacterial 2,3-butanediol production. Metabolic Engineering 22:76-82. 10.1016/j.ymben.2014.01.001

Qi FX, Yao L, Tan XM, and Lu XF. 2013. Construction, characterization and application of molecular tools for metabolic engineering of Synechocystis sp. Biotechnology Letters 35:1655-1661. 10.1007/s10529-013-1252-0

Ramey CJ, Baron-Sola A, Aucoin HR, and Boyle NR. 2015. Genome Engineering in Cyanobacteria: Where We Are and Where We Need To Go. Acs Synthetic Biology 4:1186-1196. 10.1021/acssynbio.5b00043

Ruffing AM, Jensen TJ, and Strickland LM. 2016. Genetic tools for advancement of Synechococcus sp PCC 7002 as a cyanobacterial chassis. Microbial Cell Factories 15. ARTN 190

10.1186/s12934-016-0584-6

Salis HM, Mirsky EA, and Voigt CA. 2009. Automated design of synthetic ribosome binding sites to control protein expression. Nature Biotechnology 27:946-U112. 10.1038/nbt.1568

Stanier RY, Kunisawa R, Mandel M, and Cohenbaz.G. 1971. Purification and Properties of Unicellular BlueGreen Algae (Order Cchroococcales). Bacteriological Reviews 35:171-\&.

Storch M, Casini A, Mackrow B, Fleming T, Trewhitt H, Ellis T, and Baldwin GS. 2015. BASIC: A New Biopart Assembly Standard for Idempotent Cloning Provides Accurate, Single-Tier DNA Assembly for Synthetic Biology. Acs Synthetic Biology 4:781-787. 10.1021/sb500356d

Taton A, Unglaub F, Wright NE, Zeng WY, Paz-Yepes J, Brahamsha B, Palenik B, Peterson TC, Haerizadeh F, Golden SS, and Golden JW. 2014. Broad-host-range vector system for synthetic biology and biotechnology in cyanobacteria. Nucleic Acids Research 42. ARTN e136 
460

461

462

463

464

465

466

467

468

469

470

471

472

473

474

475

476

477

478

479

480

481

482

483

484

485 10.1093/nar/gku673

Veetil VP, Angermayr SA, and Hellingwerf KJ. 2017. Ethylene production with engineered Synechocystis sp PCC 6803 strains. Microbial Cell Factories 16. ARTN 34

$10.1186 / \mathrm{s} 12934-017-0645-5$

Vuorijoki L, Isojarvi J, Kallio P, Kouvonen P, Aro EM, Corthals GL, Jones PR, and Muth-Pawlak D. 2016. Development of a Quantitative SRM-Based Proteomics Method to Study Iron Metabolism of Synechocystis sp. PCC 6803. J Proteome Res 15:266-279. 10.1021/acs.jproteome.5b00800

Wang B, Eckert C, Maness PC, and Yu JP. 2018. A Genetic Toolbox for Modulating the Expression of Heterologous Genes in the Cyanobacterium Synechocystis sp PCC 6803. Acs Synthetic Biology 7:276-286. 10.1021/acssynbio.7b00297

Wang B, Wang J, Zhang W, and Meldrum DR. 2012. Application of synthetic biology in cyanobacteria and algae. Frontiers in Microbiology 3. ARTN 344

10.3389/fmicb.2012.00344

Weissgerber TL, Milic NM, Winham SJ, and Garovic VD. 2015. Beyond bar and line graphs: time for a new data presentation paradigm. PLoS Biol 13:e1002128. 10.1371/journal.pbio.1002128

Yu Y, You L, Liu DY, Hollinshead W, Tang YJJ, and Zhang FZ. 2013. Development of Synechocystis sp PCC 6803 as a Phototrophic Cell Factory. Marine Drugs 11:2894-2916. 10.3390/md11082894

Yunus IS, and Jones PR. 2018. Photosynthesis-dependent biosynthesis of medium chain-length fatty acids and alcohols. Metabolic Engineering 49:59-68. https://doi.org/10.1016/j.ymben.2018.07.015

Zelcbuch L, Antonovsky N, Bar-Even A, Levin-Karp A, Barenholz U, Dayagi M, Liebermeister W, Flamholz A, Noor E, Amram S, Brandis A, Bareia T, Yofe I, Jubran H, and Milo R. 2013. Spanning highdimensional expression space using ribosome-binding site combinatorics. Nucleic Acids Research 41. ARTN e98

10.1093/nar/gkt151 


\section{Figure 1 (on next page)}

Measured fluorescence indicating eYFP expression levels in Synechocystis sp. PCC 6803.

Comparison of eYFP fluorescence in Synechocystis sp. PCC 6803 depending on the RBS sequence preceding the eYFP-encoding gene. Five different RBS sequences were investigated ( $A, B, C, D, E)$. The fluorescence was normalized against wild-type strain control and cell density. The horizontal line represents the mean average for each treatment, $n=3$ (biological replicates). Means without a common superscript letter differ $(\mathrm{P}<0.05)$ as analyzed by one-way ANOVA and the TUKEY test (Assaad et al, 2014). 


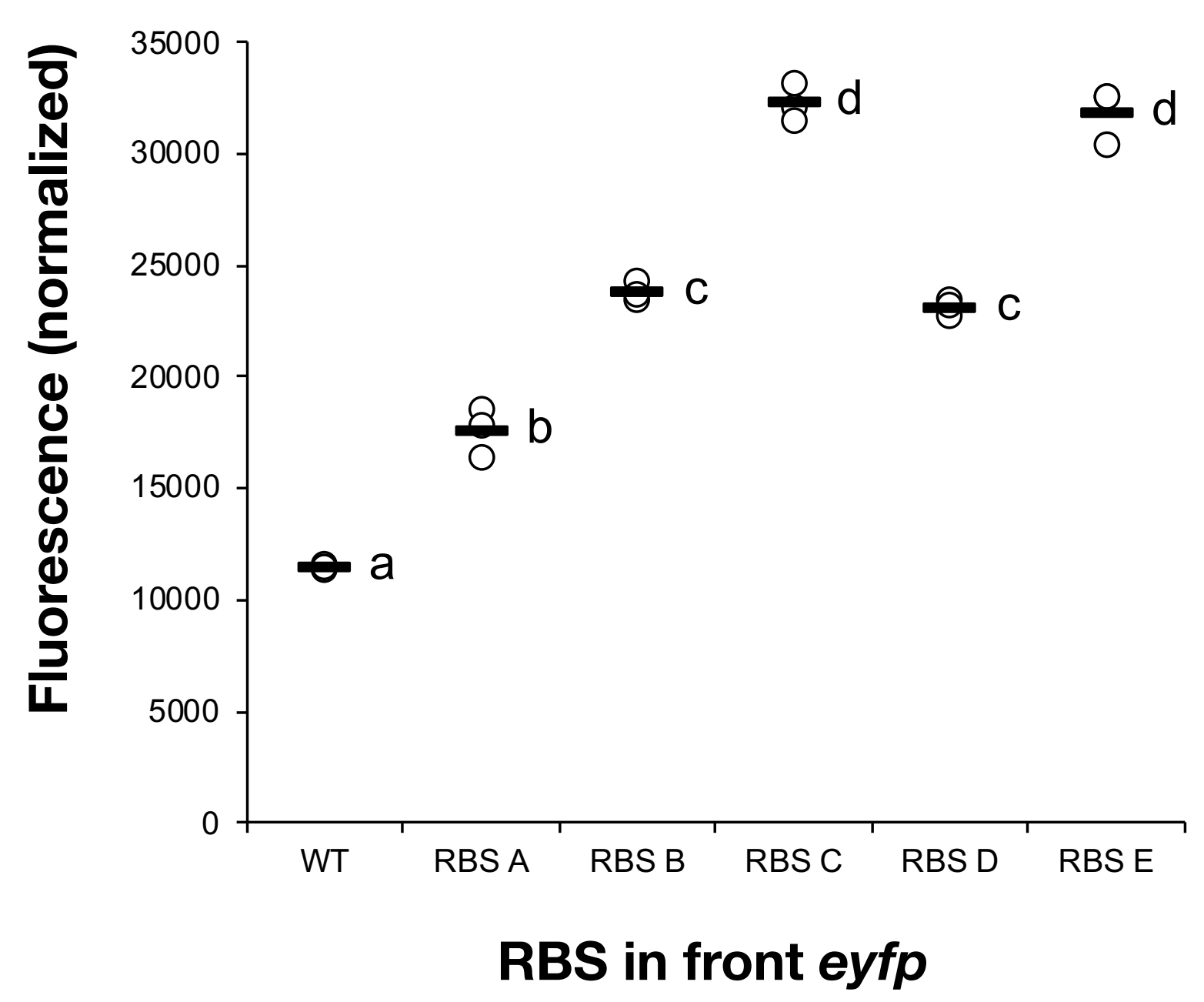




\section{Figure 2 (on next page)}

The ethanol titre in cultures of Synechocystis sp. PCC 6803 RBSx strains

The titre of ethanol relative to the optical density (g/L/OD) was measured after 7 days of cultivation of the 24 strains of Synechocystis sp. PCC 6803 with combinations of pdcand adhgenes preceded by five different ribosome binding sequences (A, B, C, D, E). The plot shows Individual measurements for all 24 strains and the wild-type strain. The horizontal line represents the mean average for each treatment. All ethanol titre values were normalized relative to each respective final OD. Means without a common superscript (A) or lowercase (B) letter differ $(P<0.05)$ as analyzed by two-way (1st RBS, 2nd RBS) ANOVA and the TUKEY test (Assaad et al, 2015). 


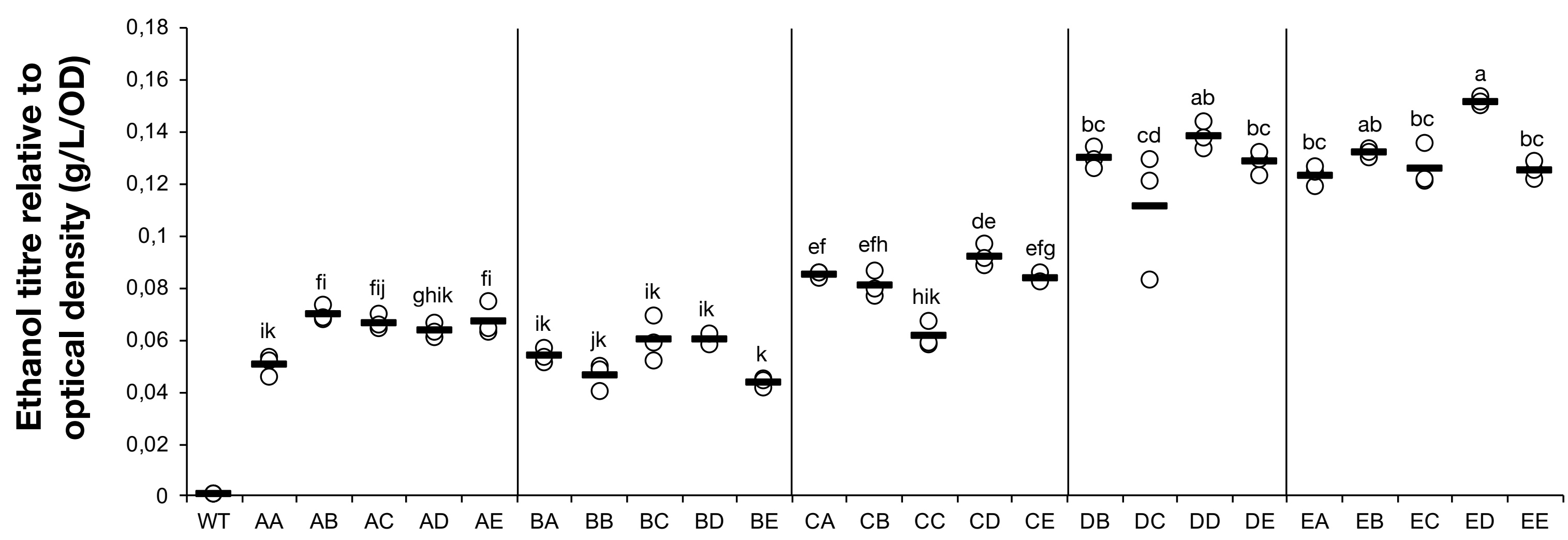

RBS in front of pdc and adh, respectively 
Figure 3 (on next page)

The relative quantity of Pdc \& Adh protein in Synechocystis sp. PCC 6803 RBSx ethanol producing strains after 7 days of cultivation

(A) Pdc and (B) Adh. The Y-axis displays the relative quantity of Pdc or Adh when normalised to the internal standard, AtpB. The horizontal line represents the mean average for each treatment. On the X-axis, the first capitalized letter stands for the RBS upstream of the pdcgene, the second capitalized letter stands for the RBS upstream of adh. The inset just above the $\mathrm{X}$-axis displays grouping of means by a common lowercase letter that do not differ $(P<0.05)$ as analyzed by two-way ANOVA and the TUKEY test (Assaad et al, 2015). WT = wild-type Synechocystis sp. PCC 6803. 
A

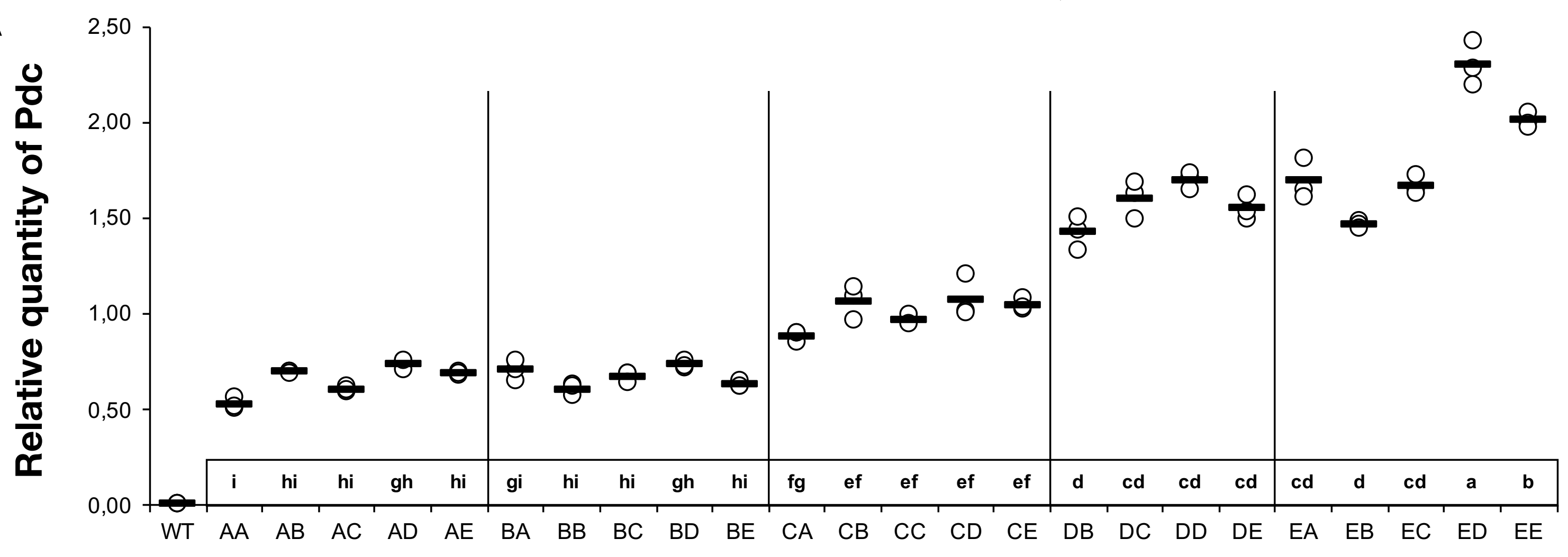

RBS in front of pdc and adh, respectively

B

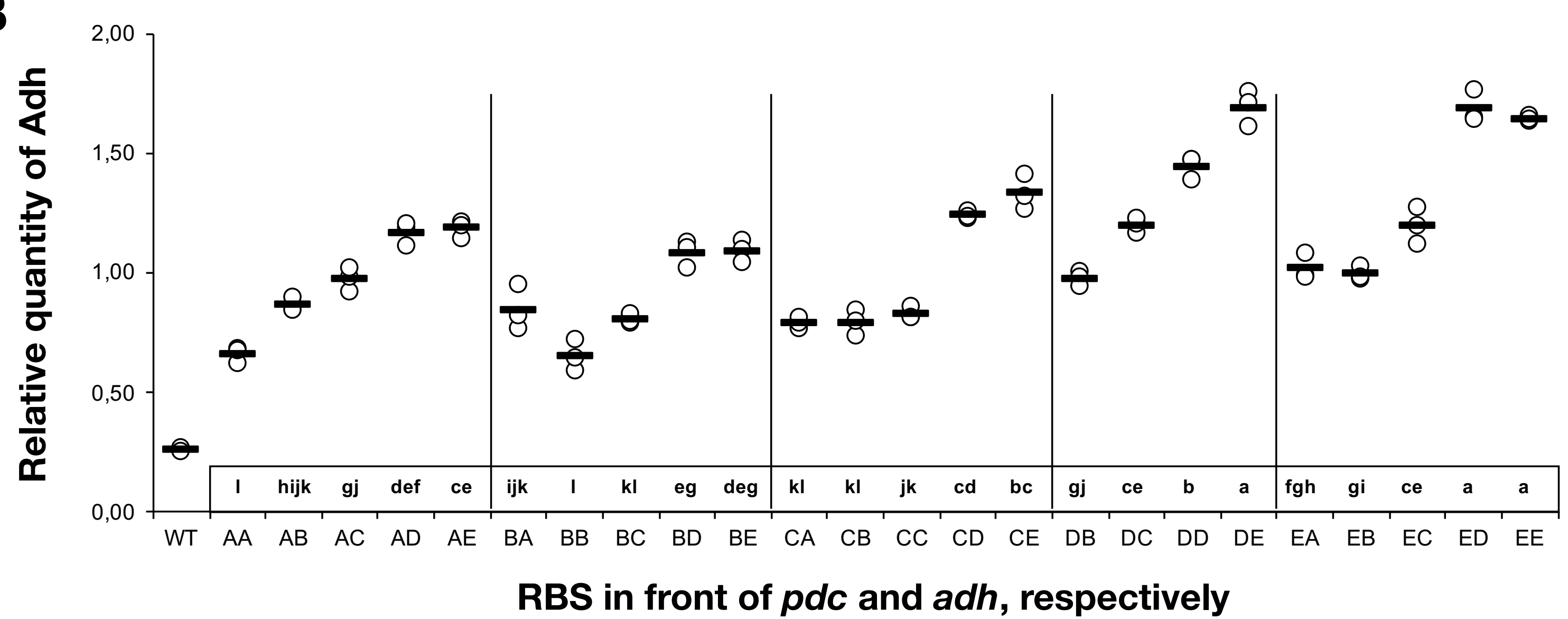


Figure 4 (on next page)

The relationship between the ethanol titre and the relative quantity of each pathway protein in Synechocystis sp. PCC 6803 RBSx ethanol producing strains after 7 days of cultivation

(A) Pdc and (B) Adh. The $R^{2}$ value was obtained from a linear fitted regression using Excel. The same data is plotted also in Figure 2 and 3. 
PeerJ

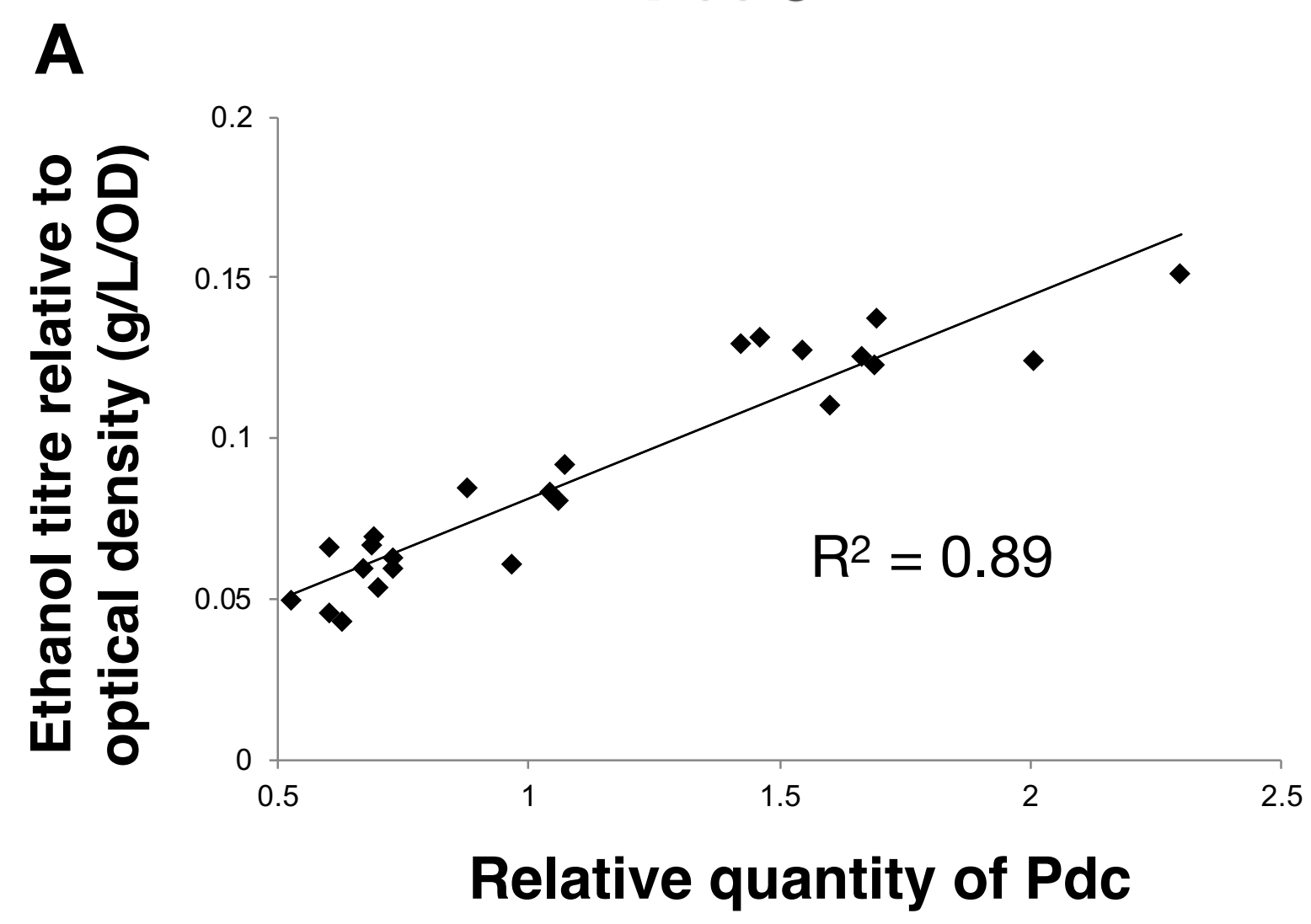

B

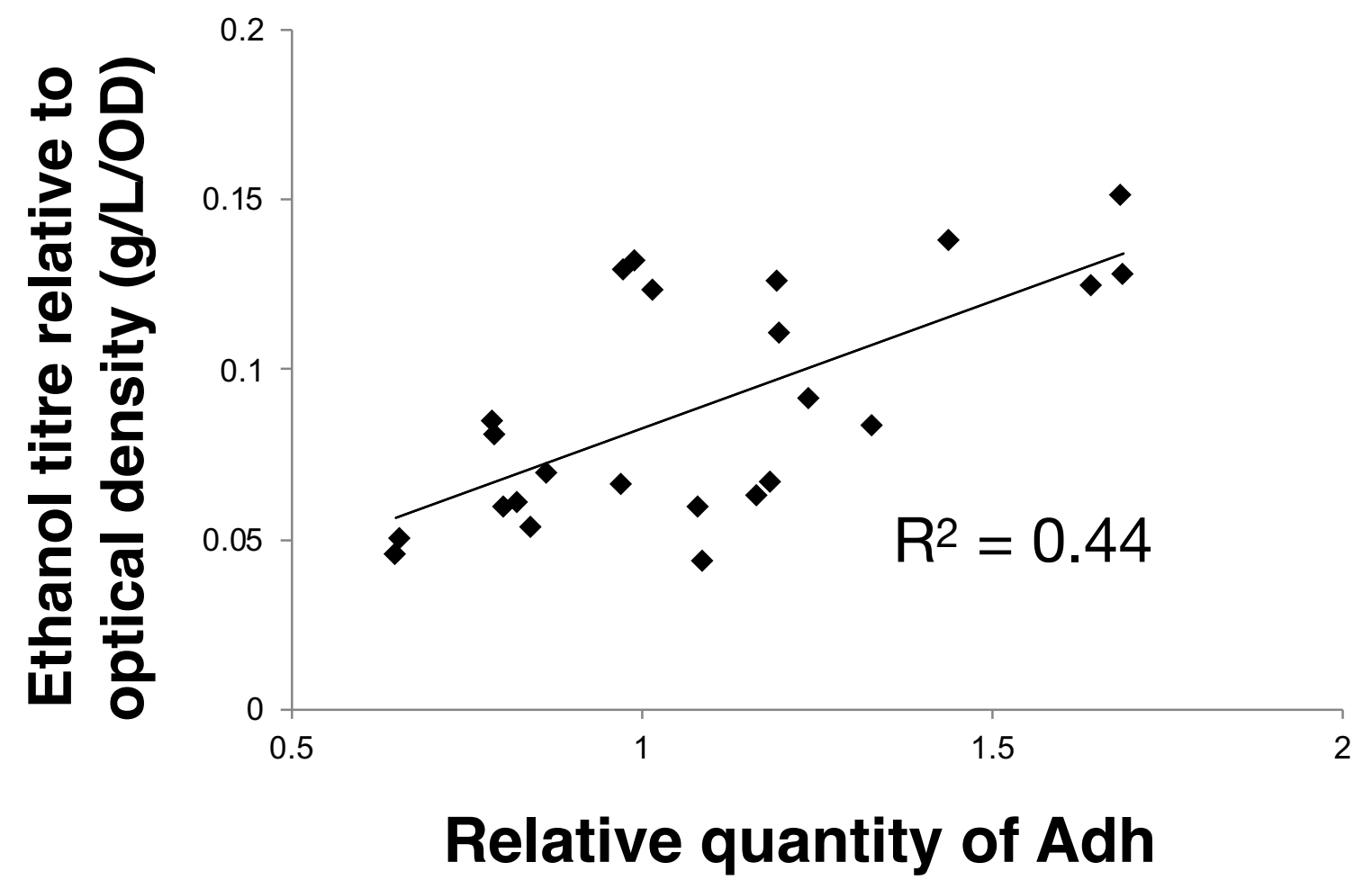




\section{Figure $\mathbf{5}$ (on next page)}

The ethanol titre and relative quantity of ethanol pathway proteins in mono- and bicistronic ethanologenic strains

The experimental treatments were 5 strains with the same ribosome binding sequence in front of both pdc and adh genes (AA, BB, CC, DD, EE) and 5 strains of Synechocystis sp. PCC 6803 harbouring only the $p d c$ gene preceded by one of five different ribosome binding sequences $(A, B, C, D, E)$. The strains were cultured for 7 days before samples were taken for measurements. (A) Ethanol titre. (B) Comparison of the expression levels of Pdc (black bars) and Adh (grey bars), normalised relative to AtpB, in Synechocystis sp. PCC 6803 strains harboring the whole ethanol cassette or the pdcgene alone. The horizontal line (in A) or bar (in B) represent the mean average for each treatment. Means without a common lowercase letter differ $(\mathrm{P}<0.05)$ as analyzed by one-way ANOVA and the TUKEY test (Assaad et al, 2014). One-way ANOVA was employed instead of two-way ANOVA in case the expression of Pdc was affected by the presence or absence of adh. (C) Correlation between ethanol titer and quantity of Pdc. On the X-axis of panels (B) and (C), the first capitalized letter stands for the RBS upstream of the pdcgene, the second capitalized letter (if present) stands for the RBS upstream ofadh. All ethanol titre values were normalized relative to each respective final OD. 


\section{Figure 6 (on next page)}

The ethanol titre, relative quantity of introduced proteins and final optical density in strains with modified central carbon metabolism enzymes

Apart from the wild-type control (WT), all strains were based on Synechocystis sp. PCC 6803 with an operon containing $p d c$ and $a d h$, both preceded by ribosome binding site $E$, integrated into the slr0168 neutral site. The experimental treatments consisted of the presence or absence of a second operon located at a different genomic locus (slr1395), in which 5 different ribosome binding sequences ( $A, B, C, D, E)$ preceded both pyk and bibp-note that strains could only be obtained with some of the combinations. The strains were cultured for 7 days before samples were taken for measurements. (A) Ethanol titre. (B) Comparison of the expression levels of Pdc (light grey), Adh (medium grey), Pyk (dark grey) and BiBP (black) normalised relative to AtpB. (C) Final optical density on day 7. The horizontal line (in A and C) or bar (in B) represent the mean average for each treatment. Means without a common lowercase letter just above the $\mathrm{X}$-axis differ $(\mathrm{P}<0.05)$ as analyzed by one-way ANOVA and the TUKEY test (Assaad et al, 2014). Independent ANOVA tests were carrie out for each of the four proteins. All ethanol titre values were normalized relative to each respective final OD. 


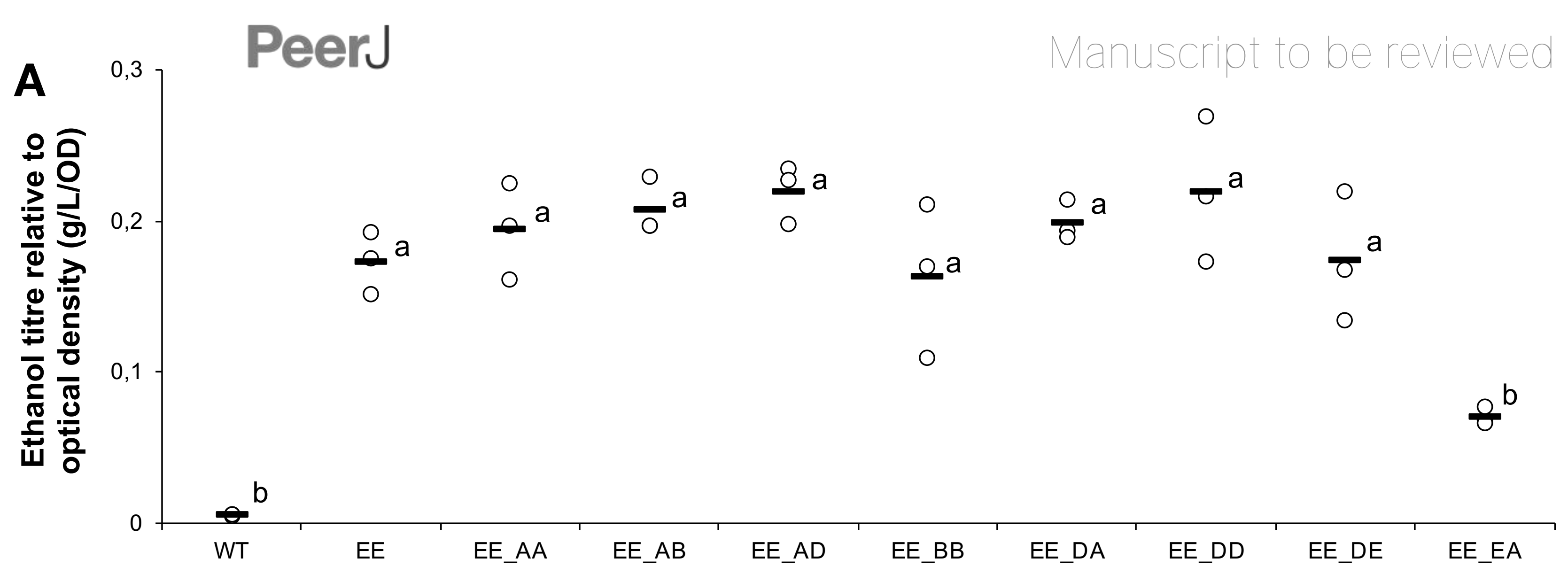

B
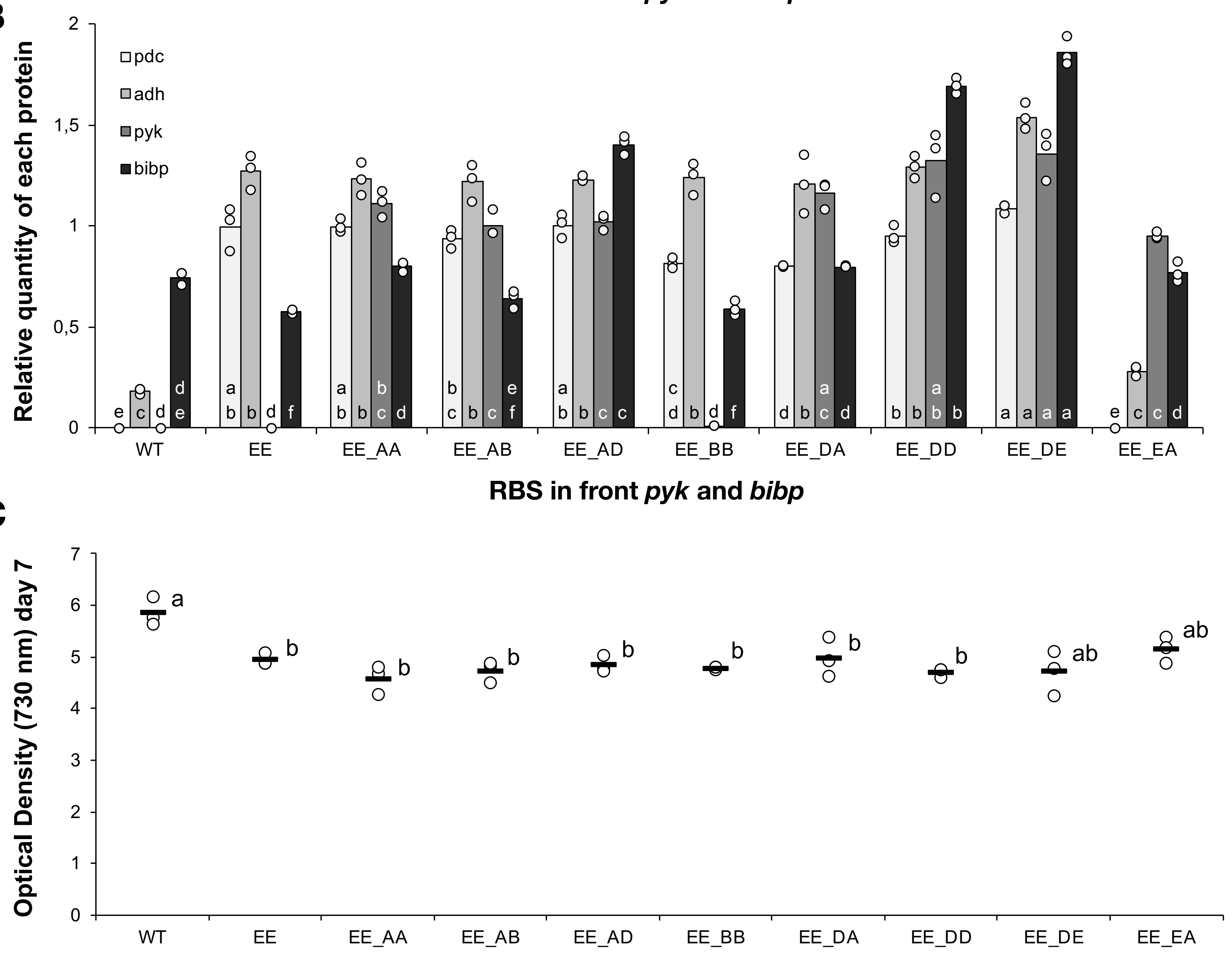